\title{
The role of hypoxia on the acquisition of epithelial- mesenchymal transition and cancer stemness: a possible link to epigenetic regulation
}

\author{
Chang Dong Yeo, Nahyeon Kang, Su Yeon Choi, Bit Na Kim, Chan Kwon Park, Jin Woo Kim, \\ Young Kyoon Kim, and Seung Joon Kim
}

Division of Pulmonology, Department of Internal Medicine, The Cancer Research Institute, College of Medicine, The Catholic University of Korea, Seoul, Korea

Received: September 29, 2016 Accepted: June 15, 2017

\section{Correspondence to Seung Joon Kim, M.D. \\ Division of Pulmonology, Department of Internal Medi- cine, College of Medicine, Seoul St. Mary's Hospital, The Catholic University of Korea, 222 Banpo- daero, Seocho-gu, Seoul 06591, Korea \\ Tel: $+82-2-2258-6063$ \\ Fax: +82-2-599-3589 \\ E-mail: cmcksj@catholic.ac.kr}

A hypoxic microenvironment leads to cancer progression and increases the metastatic potential of cancer cells within tumors via epithelial-mesenchymal transition (EMT) and cancer stemness acquisition. The hypoxic response pathway can occur under oxygen tensions of $<40 \mathrm{mmHg}$ through hypoxia-inducible factors (HIFs), which are considered key mediators in the adaptation to hypoxia. Previous studies have shown that cellular responses to hypoxia are required for EMT and cancer stemness maintenance through HIF-1 $\alpha$ and HIF-2 $\alpha$. The principal transcription factors of EMT include Twist, Snail, Slug, Sipı (Smad interacting protein 1), and ZEB1 (zinc finger E-box-binding homeobox 1). HIFs bind to hypoxia response elements within the promoter region of these genes and also target cancer stem cell-associated genes and mediate transcriptional responses to hypoxia during stem cell differentiation. Acquisition of stemness characteristics in epithelial cells can be induced by activation of the EMT process. The mechanism of these phenotypic changes includes epigenetic alterations, such as DNA methylation, histone modification, chromatin remodeling, and microRNAs. Increased expression of EMT and pluripotent genes also play a role through demethylation of their promoters. In this review, we summarize the role of hypoxia on the acquisition of EMT and cancer stemness and the possible association with epigenetic regulation, as well as their therapeutic applications.

Keywords: Hypoxia; Epithelial-mesenchymal transition; Cancer stemness; Epigenetic regulation

\section{INTRODUCTION}

The majority of tumor cells in solid tumors lack blood supply, resulting in a hypoxic tumor microenvironment [1]. Prolonged hypoxic exposure of tumor cells in a growing tumor may alter cellular behavior causing a tumor to acquire a more aggressive phenotype [2]. In recent decades, hypoxia-induced epithelial-mesenchymal transition (EMT) has been characterized in multiple hu- man cancers $[1,3-5]$.

EMT is a process by which epithelial cells are converted into mesenchymal cells through loss of cell polarity, a decrease in cell-to-cell adhesion, and a gain of migration ability [6]. EMT was first defined as an aspect of embryogenesis, but recent observations support the critical role of EMT in tumor progression and transition of an early stage tumor into an invasive malignancy with complicated and comprehensive cellular reprogramming [7]. 
The process of EMT induces mesenchymal phenotypes and self-renewal ability in cancer cells, which is believed to be associated with the gain of cancer stemness properties [8].

Cancer stemness describes a small percentage of cells with stem-like properties residing in a tumor that are treatment-resistant [9]. These cancer stem cells (CSCs) are tumor cells that possess self-renewal and multilineage differentiation capacity that can generate secondary tumors [10]. Recent studies have shown that the generation of CSCs occurs in part as a result of EMT, and that hypoxia-induced EMT leads to an increase in CSCs during tumor progression, which subsequently enhances invasion and metastasis [11]. Acquisition of EMT and cancer stemness plays a role in metastasis and treatment resistance, which are associated with poor prognosis [10].

Epigenetic regulation, including DNA methylation and histone modification, is involved in the pathogenesis of EMT and CSCs [7,12]. DNA methylation results in the addition of methyl groups at the 5'-position of cytosine residues of $\mathrm{CpG}$ dinucleotides found in mammalian DNA, which is mediated by three types of active DNA methyltransferases (DNMTs) $[12,13]$. DNA methylation plays an important role in the loss of pluripotency and developmental plasticity in cells [12]. Recently, epigenetic markers of CSCs and the associated signaling cascades have been proposed as promising therapeutic targets [12].

This review summarizes previous reports and recent advances in hypoxia-induced EMT and CSC properties, as well as their potential association with epigenetic regulation.

\section{HYPOXIA IN TUMORS}

\section{Definition of tumor hypoxia}

Inadequate perfusion and diffusion within tumors can lead to a hypoxic tumor microenvironment, which is more severe in anemic cancer patients due to a decreased $\mathrm{O}_{2}$ transport capacity [14]. Prolonged oxygen deficiency in a growing tumor can induce an aggressive cellular phenotype [2]. Ambient air is $21 \% \mathrm{O}_{2}(150 \mathrm{mmHg})$ and tissue hypoxia is typically defined as oxygen partial pressure $\leq 2 \% \mathrm{O}_{2}$ ( $\leq 15 \mathrm{mmHg}$ ). Severe hypoxia (or anoxia) is defined as $\leq 0.02 \% \mathrm{O}_{2}$. Most mammalian tissues exist at $2 \%$ to $9 \% \mathrm{O}_{2}$ (average $40 \mathrm{mmHg}$ ) [15]. Uterine cervix, head and neck, and breast cancer showed overall median $\mathrm{pO}_{2}$ of $10 \mathrm{mmHg}$ with the overall hypoxic fraction $\left(\mathrm{pO}_{2} \leq 2.5 \mathrm{mmHg}\right)$ being approximately $25 \%$. However, pancreatic adenocarcinoma had the lowest median $\mathrm{pO}_{2}$ (2 mmHg) [14]. Tumor cells can survive prolonged oxygen deficiency through adaptive cellular responses to hypoxic stress [16].

\section{Measurement of hypoxia in multiple cancers and its clinical significance}

Invasive and noninvasive procedures have been developed to assess tumor oxygenation status in xenografts and tumors [14]. The method to detect tissue oxygenation status should take into account the feasibility of the approaches for a specific experiment. The gold standard method for the detection and characterization of tumor oxygenation status is an invasive intratumoral computerized polarographic oxygen-sensitive needle electrode system, which provides a direct measurement of tissue oxygenation status [17]. Studies based on polarographic oxygen needle electrodes showed that pretherapeutic tumor hypoxia is associated with poor prognosis in locally advanced uterine cervix, head and neck cancers, and soft tissue sarcomas [18]. Nevertheless, this invasive technique has limited clinical applications. In some cases, these electrodes are too dangerous for use and cannot reach deep-seated tumors [19].

The methods to detect tissue oxygenation status have changed from direct needle-sensor oxygen measurements to indirect assays (hypoxia-inducible factor [HIF]-related changes; bioreductive metabolism), and these indirect assays can be analyzed non-invasively [19]. Surrogate markers for tumor hypoxia-related endogenous proteins (e.g., HIF-1 $\alpha$, GLUT-1 [glucose transporter 1], and CA IX [carbonic anhydrase IX]) showed prognostic significance in patient outcomes [18]. Exogenous hypoxia markers, such as 2-nitroimidazoles (e.g., pimonidazole and $\mathrm{EF}_{5}$ ), are chemically modified in hypoxic cells. However, the prognostic use of these exogenous markers appears to be limited [18].

\section{Role of the HIFs system}

Tumor hypoxia occurs when the consumption of oxygen exceeds its delivery by the vascular system [2]. Since angiogenesis is required for tumor growth, hypoxia is both induced from and results in tumorigenesis [20]. Ac- 
tivation of the hypoxic response pathway can occur under oxygen tensions $<40 \mathrm{mmHg}$ through stabilization of HIFs, which have been identified as key mediators of adaptation to hypoxia [1]. HIF-1 exists as a heterodimer that includes HIF-1 $\alpha$ and HIF-1 $\beta$ subunits. Although HIF-1 $\alpha$ is found at very low levels in a normoxic microenvironment, HIF-1 $\beta$ is ubiquitously expressed [20].

Under normoxic conditions, the availability of oxygen allows prolyl-hydroxylase domain (PHD) protein to hydroxylate proline residues on the HIF-1 $\alpha$ protein. This hydroxylation leads to a conformational change in HIF$1 \alpha$, which allows the von Hippel-Lindau (VHL) protein to recognize and bind to it. This leads to proteosomal degradation of the protein [21].

At reduced oxygen concentrations, PHD activity decreases and rapid degradation of HIF-1 $\alpha$ does not occur. Consequently, hydroxylation of HIF-1 $\alpha$ proline residues decreases, resulting in protein stabilization. HIF-1 $\alpha$ can then translocate to the nucleus and form a heterodimer with HIF-1 $\beta$, a protein also localized to the nucleus. This dimerization forms HIF-1, which binds to hypoxia response elements (HREs) and regulates angiogenesis, glycolysis, growth-factor signaling, immortalization, genetic instability, tissue invasion and metastasis, apoptosis, and $\mathrm{pH}$ regulation [22].

HIF-2 is the second member of HIF transcription factors. A dimeric complex of HIF-2 $\alpha$ and HIF-1 $\beta$ activates gene expression and initiates transcription from the HIF-1 $\alpha$ HRE [23]. Under hypoxia, PHD activity is inhibited, the binding of VHL is prevented, and HIF-2 $\alpha$ enters the nucleus to heterodimerize with HIF-1 $\beta$. Subsequently, HIF-2 $\alpha$ binds to HRE and transactivates a variety of hypoxia response genes. During chronic hypoxic conditions, HIF-2 performs a crucial role in driving the hypoxic response, whereas HIF-1 drives the initial response to hypoxia [24]. Although HIF-1 and HIF-2 share many transcriptional targets, HIF-1 commonly controls metabolism and glycolysis, whereas HIF-2 may play a more oncogenic role [1].

\section{HYPOXIA-INDUCED EMT AND ACQUISITION OF STEM CELL PROPERTIES}

\section{Hypoxia and EMT in tumorigenesis}

EMT is induced by hypoxia and other signaling path- ways, including Notch, transforming growth factor $\beta$ (TGF- $\beta$ ), Sonic Hedgehog, and Wnt signaling [25]. Hypoxia-induced EMT is involved in cancer progression and metastasis, and HIF-1 has been identified as a regulator of EMT in human cancers. Phenotypic changes in EMT include the absence of epithelial markers (e.g., E-cadherin) and up-regulation of mesenchymal markers (e.g., vimentin, fibronectin, and N-cadherin) [6]. EMT can be modulated by HIF, which regulates the expression and functional activity of principal transcription factors including Twist, Snail, Slug, Sipı (Smad interacting protein 1), and zinc finger E-box-binding homeobox 1 (ZEB1) [26]. The promoter region of Twist contains an HRE, which can be bound by HIF. Hypoxic microenvironments induce EMT master regulators that promote EMT changes, such as E-cadherin attenuation through Snail up-regulation, as well as matrix metalloproteinase (MMP) production through activation of Slug [27]. In patients with resected non-small cell lung cancer, co-expression of HIF-1 $\alpha$, Twist1, and Snail was a significant predictor of poor prognosis [28]. In addition, inflammatory cells including neutrophils, lymphocytes, macrophages, and myeloid-derived suppressor cells, which secrete inflammatory cytokines including tumor necrosis factor $\alpha$, TGF- $\beta$, interleukin 1 (IL-1), IL-6, and IL-8, may play major roles in hypoxia-induced EMT [26]. TGF- $\beta$ production stimulated by hypoxic stress from myeloid cells, mesenchymal cells, and cancer cells provides a possible mechanism through which hypoxia induces TGF- $\beta$ triggered EMT [26,29].

\section{Normal stem cells and cancer stem cells}

Stem cells are defined as undifferentiated biological cells that can replicate themselves via self-renewal and produce specialized cells of particular tissues or organs through differentiation [9,30]. There are three broad types of stem cells: embryonic stem cells, mesenchymal stem cells, and induced pluripotent stem cells. Embryonic stem cells are isolated from the inner cell mass of blastocysts, mesenchymal stem cells are obtained from adult adipose tissue, blood, bone marrow, and cord blood, and induced pluripotent stem cells can now be prepared through advanced technology (viral and episomal vector-mediated reprogramming, as well as microRNA- and protein-mediated induction of pluripotency) [30,31]. 
CSCs are cancer cells found within tumors or hematological cancers that possess characteristics associated with normal stem cells, including the capacity to selfrenew and cause heterogeneous lineages of cancer cells that comprise the tumor [32]. These cells persist in tumors as a distinct population and can lead to treatment resistance, as well as relapse and metastasis by giving rise to new tumors. Hematologic malignancies, such as leukemia and multiple myeloma, have been characterized previously, showing that only a small subset of cancer cells are capable of extensive proliferation. It has also been shown in solid tumors that only a small number of cancer cells in lung, ovarian, or neuroblastoma cancer are actually tumorigenic, and these tumorigenic cells are considered to be CSCs $[9,33]$. Therefore, CSCs are believed to be tumor-initiating cells that are resistant to chemotherapy and radiation therapy [34].

Airway injury and epithelial cell loss following damage may regulate stem or progenitor cell-mediated repair in basal cells, Clara cells, alveolar type II cells, and cells in the bronchioalveolar duct junction [35]. These cells may be the origin of CSCs, which are important for CSC research $[1,36]$.

\section{Hypoxia and the acquisition of stem cell traits}

CSC biomarkers can be categorized as stem cell-like markers (CD133, CD44, $\mathrm{CD}_{34}$, and $\mathrm{CD}_{24}$ ), pluripotency genes (Oct3/4, Nanog, sex determining region Y-box 2 [SOX2], and $\mathrm{MYC}$ ), invasiveness markers (vimentin, $\mathrm{N}$-cadherin, Snail, Twist, and ZEB1), and markers of drug resistance (aldehyde dehydrogenase and ATP-binding cassette $[\mathrm{ABC}]$ transporters) [12]. The CD24+ cell population expresses pluripotent genes including $\mathrm{SOX}_{2}$, octamer-binding transcription factor 4 (Oct4), Nanog, B lymphoma Mo-MLV insertion region 1 homolog (Bmi-1), Rex-1, and the stimulated stem-cell specific Wnt/ $\beta$-catenin signaling pathway, which increases resistance to chemotherapeutic drugs [37].

HIFs target CSC-associated genes [16] and mediate transcriptional responses to hypoxia during normal embryonic development, tumor growth, and stem cell differentiation. Hypoxia promotes self-renewal capability and inhibits differentiation in glioma stem cells [38]. Reduction in the oxygen level in human glioma cell cultures significantly increases the percentage of cells expressing CD133 [39]. Expression of HIF-1 and lactate dehydrogenase 5 in tumor tissues is associated with CD44-enriched stem cell populations, which is a poor prognostic factor in bladder cancer patients treated with radiotherapy [40]. Previous studies have shown that cellular responses to hypoxia are required for CSC maintenance through HIF-1 $\alpha$ and HIF-2 $\alpha$, but that HIF-2 $\alpha$ is more specific and selective for glioblastoma stem cells [41].

C-X-C chemokine receptor type 4 (CXCR4), an $\alpha$-chemokine receptor specific for SDF-1 (stromal cell-derived factor 1), induces downstream signaling through several different pathways in tumorigenesis and progression $[42,43]$. In the Lewis lung carcinoma cell line, CXCR4+ cells show cancer metastatic stem cell properties [44]. In colon cancer, hypoxia promotes the expression of CXCR4 transcript and protein at the cell membrane, which is regulated by HIF-1 $\alpha$ [45]. Liang et al. [46] reported that CXCR4/C-X-C motif chemokine 12 (CXCL12) induces Akt phosphorylation, which stimulates expression of vascular endothelial growth factor (VEGF). These reports suggest that the CXCR4/CXCL12 axis induces angiogenesis, regulates EMT, and promotes tumor progression and metastasis [42].

\section{LINKING EMT TO CSC PROPERTIES AND THEIR EPIGENETIC REGULATION}

\section{EMT-induced cancer stemness acquisition}

Fig. 1 summarizes the overall cellular process of downstream signaling induced by a hypoxic microenvironment. Acquisition of stemness characteristics in epithelial cells can be induced by activation of the EMT process [47]. Human mammary epithelial cells that undergo EMT can form mammospheres, soft agar colonies, and tumors [48]. In a mammary tumor progression model, Morel et al. [49] showed that cancer cells possessing both stemness and tumorigenic characteristics of CSCs can be derived by EMT induction. Moreover, the early events of cellular invasion in situ can facilitate cancer dissemination, in part through EMT [50].

Factors that control EMT also play a role in stemness in the context of cancer [47]. Snail and Slug are transcription factors involved in wound healing or protecting stem cell/progenitor cells from DNA damage. In ovarian cancer cells, Snail and Slug mediate the acquisition 


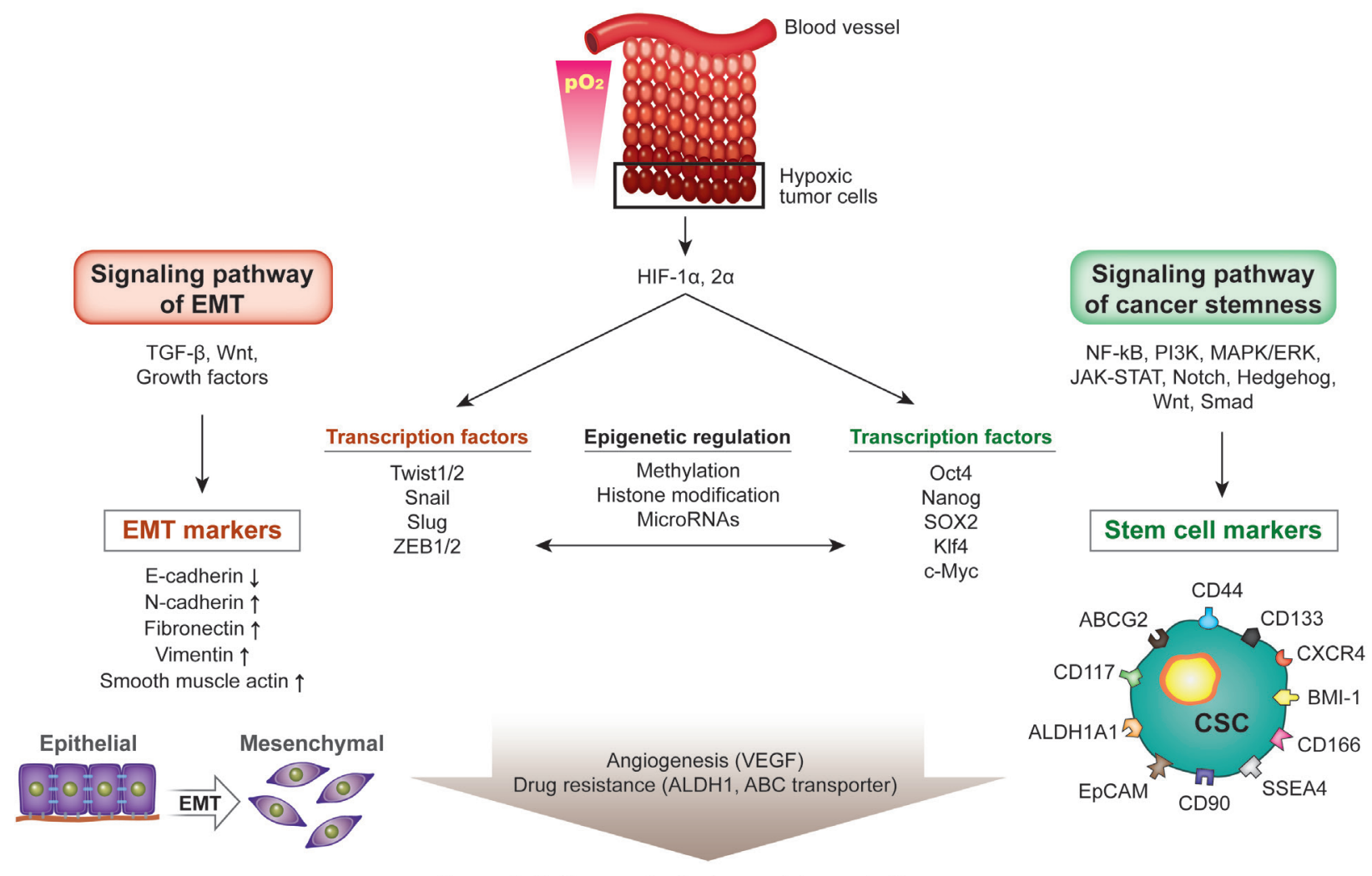

Tumor initiation, metastasis, resistance to therapy

Figure 1. Schematic representation of the downstream signaling cascade initiated by a hypoxic microenvironment. Hypoxic response pathways result in cancer cells acquiring epithelial-mesenchymal transition (EMT) and stemness through hypoxia-inducible factor $1 \alpha(\mathrm{HIF}-1 \alpha)$ and HIF-2 $\alpha$. These phenotypic changes are regulated by epigenetic alterations. The EMT signaling pathway is primarily mediated by transforming growth factor $\beta$ (TGF- $\beta$ ), Wnt, and other growth factors including endothelial growth factor (EGF), hepatocyte growth factor (HGF), insulin-like growth factor (IGF), and fibroblast growth factor (FGF) families. The primary transcription factors of EMT are Twistı/2, Snail, Slug, and zinc finger E-box-binding homeobox 1/2 (ZEB1/2). HIFs also target cancer stem cell (CSC)-associated signaling pathways and mediate transcriptional responses to hypoxia. Acquisition of stemness characteristics in epithelial cells can be induced by activation of the EMT process. Ultimately, cancer cells show tumor initiation, metastasis, and resistance to therapy. Oct4, octamer-binding transcription factor 4; SOX2, sex determining region Y-box 2; Klf4, Kruppel-like factor 4; NF- $\mathrm{B}$, nuclear factor $\kappa \mathrm{B}$; PI $3 \mathrm{~K}$, phosphoinositide 3-kinase; MAPK/ ERK, mitogen-activated protein kinase/extracellular signal-related kinase; JAK-STAT, Janus kinase-signal transducer and activator of transcription; VEGF, vascular endothelial growth factor; ALDH1, aldehyde dehydrogenase 1 family; ABC, ATP-binding cassette; CD, cluster of differentiation; ABCG2, ATP-binding cassette sub-family G member 2; ALDH1A1, aldehyde dehydrogenase 1 family, member A1; EpCAM, epithelial cell adhesion molecule; SSEA4, stage-specific embryonic antigen-4; CXCR4, C-X-C chemokine receptor type 4; BMI-1, B lymphoma Mo-MLV insertion region 1 homolog.

of stem cell characteristics including radiotherapy and chemotherapy resistance, which may contribute to aggressive cancer metastasis [51]. ZEB1 is an EMT inducer and a crucial promoter of invasion and metastasis in cancer. ZEBı links EMT activation and stemness maintenance by suppressing the microRNA-200 family, which strongly induces epithelial differentiation, as well as by repressing stemness-inhibiting microRNA-203 [52]. Furthermore, stemness-related genes have been implicated in the acquisition of mesenchymal phenotypes.
In hepatocellular carcinoma patients, CD44 overexpression is associated with decreased expression of E-cadherin, increased expression of vimentin, a high level of phospho-Smad2-positive nuclei, and poor overall survival [53]. CD133, a CSC marker in multiple malignancies, contributes to EMT induction and tumor invasiveness in vitro, which is mediated by nuclear factor $\kappa \mathrm{B}$ activation, as well as stemness properties in vivo in pancreatic cancer [54]. 


\section{Effect of demethylation (or epigenetic regulation) on EMT markers}

Acquired epigenetic alterations in gene expression play a role in the early stages of carcinogenesis. These can generally be classified as DNA methylation, histone modification, chromatin remodeling, and microRNAs [55]. Epigenetic programming can regulate EMT, and EMT may be reversed by dynamic epigenetic modifications [55].

DNA methylation occurs at the 5'-position of cytosine residues within $\mathrm{CpG}$ dinucleotides and leads to gene silencing. Three types of DNMTs (DNMT1, DNMT3A, and $\mathrm{DNMT}_{3} \mathrm{~B}$ ) are responsible for this modification. DNMT1, which is a maintenance methyltranferase, maintains pre-existing methylation patterns by adding methyl groups to the hemimethylated DNA during the S-phase of the cell cycle. DNMT3 3 A and DNMT3 B, which are de novo methyltransferases, determine the methylation pattern of genes by targeting unmethylated CpG sites [12].

Promoter methylation of E-cadherin, as opposed to mutational inactivation, results in an increased mesenchymal phenotype and invasiveness in breast cancer cells [56]. Chen et al. [57] showed that DNA methylation regulates the transcription of Snail and Slug genes, and their transcription in EMT/mesenchymal epithelial transition processes are associated with the DNA methylation level in the first intron region. Treatment with 5-aza-2'-deoxycytidine, an inhibitor of DNMT, can induce expression of these Snail and Slug genes. The methylation patterns in $\mathrm{CpG}$ islands are also associated with tumor prognosis in patients with acute myeloid leukemia [58].

MicroRNAs, small non-coding RNA molecules, have also been associated with EMT. The microRNA-200 family (strong inducers of epithelial differentiation) is repressed by the EMT activator ZEB1 [52], and the tumor suppressor p53 plays an essential role in regulating EMT by modulating microRNAs [59]. Loss of p53 in mammary epithelial cells results in decreased expression of microRNA-200c and activation of the EMT process. In addition, cells that undergo EMT are often methylated in the promoter region of microRNA-203 [60]. Restoring microRNA-203 expression can compromise tumor initiation and metastasis, as well as cellular migration and invasion [6o].

\section{Effect of demethylation (or epigenetic regulation) on stem cell markers}

CSCs can be generated from epigenetic reprogramming where stem cell-specific genes regain their pluripotency, whereas genes specific for differentiation are downregulated [12]. The increased expression of pluripotent genes coincides with their promoter demethylation, which suggests that demethylation of promoter DNA may be important in the epigenetic reprogramming of somatic cell nuclei [61]. Recently, El Helou et al. [62] observed hypomethylation of breast CSCs in 68 differentially methylated regions compared with non-breast CSC populations, which worsen clinical outcomes.

The Oct4 gene, which encodes the POU-domain DNA-binding protein, is a critical pluripotency regulator. Expression of the Oct4 gene is important for the acquisition of pluripotency in embryonic cells. The Oct4 promoter in cloned cumulus cell embryos becomes gradually demethylated during nuclear reprogramming [63]. Inflammatory reactions in the cancer microenvironment can increase p53 and p21 DNA hypermethylation through DNMT1 overexpression. Upregulation of DNMT1 led to cancer initiation and CSC proliferation by downregulating p53 and p21 through DNA hypermethylation [64].

The CXCR4/CXCL12 interactions mediate maintenance and activation of cancer stemness properties [42]. Silencing of CXCL12 due to aberrant methylation is common and is an unfavorable prognostic marker in patients with lung cancer [65]. In addition, aberrant methylation of CXCL12 plays an important role in organ-specific patterns of metastasis in gastric cancer [66] and breast cancer [67]. Kubarek and Jagodzinski [68] reported that upregulation of CXCR4 and CXCL12 in endometrial cancer is mediated by DNA demethylation of CpG island promoters, which is associated with the temporary increase in $\mathrm{DNMT}_{3} \mathrm{~B} 4$. In pancreatic cancer cells, RNA interference-mediated knockdown of DNMT1 and $\mathrm{DNMT}_{3} \mathrm{~B}$ can increase promoter demethylation, which increases CXCR4 expression. These results indicate that DNMTs may be a useful therapeutic target [69].

MicroRNAs are important in the epigenetic reprogramming of CSCs [8]. MicroRNA-3O2 is believed to mediate pluripotent stem cell reprogramming and improves drug sensitivity in hepatocellular carcinoma cells [70]. MicroRNA-34a is a key negative regulator of 
CD44+ prostate cancer cells, and increased expression of microRNA-34a inhibits clonogenic expansion, tumor regeneration, and metastasis [71]. MicroRNA-200c is known to suppress BMI-1, a regulator of stem cell self-renewal, and inhibit the clonal expansion of breast cancer cells, the growth of embryonal carcinoma cells in vitro and tumor formation driven by human breast CSCs in vivo [72]. Suppression of microRNA-20o is required for normal stem cells to form mammary ducts and breast CSCs for tumor formation. In addition, overexpression of microRNA-9 was observed in breast tumors showing the breast CSC phenotype (CD44+/CD24-) and expression of EMT markers (vimentin expression and E-cadherin loss) [73]. Furthermore, it is believed that microRNA-9 inhibits the expression of CXCR4 mediated by the Wnt $/ \beta$-catenin signaling pathway in oral squamous cell carcinoma [74]. The expression of CXCR4, MMP-2, MMP-9, and VEGF are suppressed by microRNA-451, which acts as a tumor suppressor in A549 lung cancer cells [75]. Therefore, these microRNAs play an important role in controlling cancer progression and stem cell characteristics.

\section{THERAPEUTIC APPROACHES THROUGH THE EPIGENETIC REGULATION OF EMT AND CSC CHARACTERISTICS}

Both EMT and stemness are associated with resistance to chemotherapy in tumors [47]. ABC transporter genes involved in chemoresistance can be upregulated by EMT-inducing transcription factors, such as Twist, Snail, and Slug, through their ability to induce EMT [47]. The EMT process plays a role in generating the stem cell phenotype, and expression of $\mathrm{CD}_{44}$ or $\mathrm{CD}_{133}$ stemness genes confers chemoresistance [76]. CSCs express several $\mathrm{ABC}$ transporters, including $\mathrm{ABCB} 1$ (P-glycoprotein), a product of the multidrug resistance gene found in more than half of drug-resistant tumors [12].

DNMT and/or histone deacetylase (HDAC) inhibitors are believed to have therapeutic potential for chemosensitization of drug-resistant cells. The compounds 5-azaCR and 5-aza-CdR have now received US Food and Drug Administration approval for the treatment of myelodysplastic syndrome. These agents may transform highly invasive cancer cells into epithelial cells by restoring the expression of E-cadherin in various cancers [55,77]. Several HDAC inhibitors are being evaluated in clinical trials to specifically target cancer cells.

The regulatory role of microRNAs in drug-resistance has been shown in CSCs. Increasing the expression of tumor suppressor microRNAs, including the microRNA-200 family, microRNA-153, microRNA-145, microRNA-96, microRNA-34, microRNA-7, and Let-7a are used therapeutically. In addition, reduced expression of onco-microRNAs, such as microRNA-261, microRNA-181, and microRNA-21, are novel agents in cancer therapy [8]. Restoration of microRNA-200 and Let-7 family members is expected to influence and reverse EMT and the stemness phenotype, which could induce epithelial differentiation and repress the self-renewal capacity [78]. Recently, checkpoint inhibitors including anti-cytotoxic T-lymphocyte-associated protein 4 (CTLA-4), anti-programmed cell death protein 1 (anti-PD-1), and anti-PD-L1 have been proposed as promising treatments. Inhibition of CXCR4 following treatment with sorafenib in hepatocellular carcinoma has been shown to inhibit tumor growth, reduce lung metastasis, and improve survival. Furthermore, anti-PD-1 treatment showed additional antitumor activity when combined with sorafenib and the CXCR4 inhibitor [79]. Thus, epigenetic therapy alone or in combination with chemotherapy may represent a novel strategy for the treatment of drug-resistant tumors.

\section{CONCLUSIONS}

In conclusion, the association among tumor hypoxia, EMT and cancer stemness is an ongoing hot issue in the laboratory, although limitations of clinical application must be taken into consideration. Hypoxic response pathways lead cancer cells to acquire EMT and stemness and these changes are regulated by epigenetic alterations (DNA methylation, histone modification, and microRNAs). Clinical approaches through epigenetic regulation of EMT and CSC have therapeutic potentials to induce epithelial differentiation and repress self-renewal capacity, which is promising for treatment of drug-resistant tumor. 


\section{Conflict of interest}

No potential conflict of interest relevant to this article was reported.

\section{Acknowledgments}

This work was supported by a National Research Foundation of Korea (NRF) grant funded by the Korea government (MSIP) (No. 2014R1A2A1A11052422).

\section{REFERENCES}

1. Foster JG, Wong SC, Sharp TV. The hypoxic tumor microenvironment: driving the tumorigenesis of non-smallcell lung cancer. Future Oncol 2014;10:2659-2674.

2. Hockel M, Vaupel P. Tumor hypoxia: definitions and current clinical, biologic, and molecular aspects. J Natl Cancer Inst 2001;93:266-276.

3. Gao T, Li JZ, Lu Y, et al. The mechanism between epithelial mesenchymal transition in breast cancer and hypoxia microenvironment. Biomed Pharmacother 2016;80:393405 .

4. Li M, Wang YX, Luo Y, et al. Hypoxia inducible factor-1 $\alpha$ dependent epithelial to mesenchymal transition under hypoxic conditions in prostate cancer cells. Oncol Rep 2016;36:521-527.

5. Ye LY, Chen W, Bai XL, et al. Hypoxia-induced epithelialto-mesenchymal transition in hepatocellular carcinoma induces an immunosuppressive tumor microenvironment to promote metastasis. Cancer Res 2016;76:818-830.

6. Thiery JP, Acloque H, Huang RY, Nieto MA. Epithelialmesenchymal transitions in development and disease. Cell 2009;139:871-890.

7. Li L, Li W. Epithelial-mesenchymal transition in human cancer: comprehensive reprogramming of metabolism, epigenetics, and differentiation. Pharmacol Ther 2015;150:33-46.

8. Garg M. Targeting microRNAs in epithelial-to-mesenchymal transition-induced cancer stem cells: therapeutic approaches in cancer. Expert Opin Ther Targets 2015;19:285297.

9. Reya T, Morrison SJ, Clarke MF, Weissman IL. Stem cells, cancer, and cancer stem cells. Nature 2001;414:105-111.

10. Medema JP. Cancer stem cells: the challenges ahead. Nat Cell Biol 2013;15:338-344.

11. Mimeault M, Batra SK. Hypoxia-inducing factors as master regulators of stemness properties and altered metabolism of cancer- and metastasis-initiating cells. J Cell Mol Med 2013;17:30-54.

12. Shukla S, Meeran SM. Epigenetics of cancer stem cells: pathways and therapeutics. Biochim Biophys Acta 2014;1840:3494-3502.

13. Jones PA. Functions of DNA methylation: islands, start sites, gene bodies and beyond. Nat Rev Genet 2012;13:484492.

14. Vaupel P, Hockel M, Mayer A. Detection and characterization of tumor hypoxia using $\mathrm{pO}_{2}$ histography. Antioxid Redox Signal 2007;9:1221-1235.

15. Bertout JA, Patel SA, Simon MC. The impact of $\mathrm{O}_{2}$ availability on human cancer. Nat Rev Cancer 2008;8:967-975.

16. Myszczyszyn A, Czarnecka AM, Matak D, et al. The role of hypoxia and cancer stem cells in renal cell carcinoma pathogenesis. Stem Cell Rev 2015;11:919-943.

17. Yeh KA, Biade S, Lanciano RM, et al. Polarographic needle electrode measurements of oxygen in rat prostate carcinomas: accuracy and reproducibility. Int J Radiat Oncol Biol Phys 1995;33:111-118.

18. Vaupel P, Mayer A. Hypoxia in cancer: significance and impact on clinical outcome. Cancer Metastasis Rev 2007;26:225-239.

19. Koch CJ, Evans SM. Optimizing hypoxia detection and treatment strategies. Semin Nucl Med 2015;45:163-176.

20. Shimoda LA, Semenza GL. HIF and the lung: role of hypoxia-inducible factors in pulmonary development and disease. Am J Respir Crit Care Med 2011;183:152-156.

21. Sharp FR, Bernaudin M. HIF1 and oxygen sensing in the brain. Nat Rev Neurosci 2004;5:437-448.

22. Harris AL. Hypoxia: a key regulatory factor in tumour growth. Nat Rev Cancer 2002;2:38-47.

23. Gordan JD, Simon MC. Hypoxia-inducible factors: central regulators of the tumor phenotype. Curr Opin Genet Dev 2007;17:71-77.

24. Zhao J, Du F, Luo Y, Shen G, Zheng F, Xu B. The emerging role of hypoxia-inducible factor-2 involved in chemo/ radioresistance in solid tumors. Cancer Treat Rev 2015;41:623-633.

25. Zhang J, Tian XJ, Xing J. Signal transduction pathways of EMT induced by TGF-beta, SHH, and WNT and their crosstalks. J Clin Med 2016;5:41. https://doi.org/10.3390/ jem5040041.

26. Jiang J, Tang YL, Liang XH. EMT: a new vision of hypoxia promoting cancer progression. Cancer Biol Ther 
2011;11:714-723.

27. Imai T, Horiuchi A, Wang C, et al. Hypoxia attenuates the expression of E-cadherin via up-regulation of SNAIL in ovarian carcinoma cells. Am J Pathol 2003;163:1437-1447.

28. Hung JJ, Yang MH, Hsu HS, Hsu WH, Liu JS, Wu KJ. Prognostic significance of hypoxia-inducible factorıalpha, TWIST1 and Snail expression in resectable nonsmall cell lung cancer. Thorax 2009;64:1082-1089.

29. Nishi H, Nakada T, Hokamura M, et al. Hypoxia-inducible factor-1 transactivates transforming growth factorbeta3 in trophoblast. Endocrinology 2004;145:4113-4118.

30. Diecke S, Jung SM, Lee J, Ju JH. Recent technological updates and clinical applications of induced pluripotent stem cells. Korean J Intern Med 2014;29:547-557.

31. Kim N, Cho SG. Clinical applications of mesenchymal stem cells. Korean J Intern Med 2013;28:387-402.

32. Clarke MF, Dick JE, Dirks PB, et al. Cancer stem cells: perspectives on current status and future directions: AACR Workshop on cancer stem cells. Cancer Res 2006;66:9339-9344.

33. Hamburger AW, Salmon SE. Primary bioassay of human tumor stem cells. Science 1977;197:461-463.

34. Sourisseau T, Hassan KA, Wistuba I, et al. Lung cancer stem cell: fancy conceptual model of tumor biology or cornerstone of a forthcoming therapeutic breakthrough? J Thorac Oncol 2014;9:7-17.

35. Giangreco A, Arwert EN, Rosewell IR, Snyder J, Watt FM, Stripp BR. Stem cells are dispensable for lung homeostasis but restore airways after injury. Proc Natl Acad Sci U S A 2009;106:9286-9291.

36. Akunuru S, James Zhai Q, Zheng Y. Non-small cell lung cancer stem/progenitor cells are enriched in multiple distinct phenotypic subpopulations and exhibit plasticity. Cell Death Dis 2012;3:e352.

37. Yang CH, Wang HL, Lin YS, et al. Identification of CD24 as a cancer stem cell marker in human nasopharyngeal carcinoma. PLoS One 2014;9:e99412.

38. Li Z, Bao S, Wu Q, et al. Hypoxia-inducible factors regulate tumorigenic capacity of glioma stem cells. Cancer Cell 2009;15:501-513.

39. McCord AM, Jamal M, Shankavaram UT, Lang FF, Camphausen K, Tofilon PJ. Physiologic oxygen concentration enhances the stem-like properties of CD133+ human glioblastoma cells in vitro. Mol Cancer Res 2009;7:489-497.

40. Koukourakis MI, Kakouratos C, Kalamida D, et al. Hypoxia-inducible proteins HIF $1 \alpha$ and lactate dehydrogenase
$\mathrm{LDH}_{5}$, key markers of anaerobic metabolism, relate with stem cell markers and poor post-radiotherapy outcome in bladder cancer. Int J Radiat Biol 2016;92:353-363.

41. Seidel S, Garvalov BK, Wirta V, et al. A hypoxic niche regulates glioblastoma stem cells through hypoxia inducible factor 2 alpha. Brain 2010;133:983-995.

42. Wang Z, Sun J, Feng Y, Tian X, Wang B, Zhou Y. Oncogenic roles and drug target of CXCR4/CXCL12 axis in lung cancer and cancer stem cell. Tumour Biol 2016;37:8515-8528.

43. Cho BS, Kim HJ, Konopleva M. Targeting the CXCL12/ $\mathrm{CXCR}_{4}$ axis in acute myeloid leukemia: from bench to bedside. Korean J Intern Med 2017;32:248-257.

44. Nian WQ, Chen FL, Ao XJ, Chen ZT. CXCR4 positive cells from Lewis lung carcinoma cell line have cancer metastatic stem cell characteristics. Mol Cell Biochem 2011;355:241-248.

45. Romain B, Hachet-Haas M, Rohr S, et al. Hypoxia differentially regulated $\mathrm{CXCR}_{4}$ and $\mathrm{CXCR}_{7}$ signaling in colon cancer. Mol Cancer 2014;13:58.

46. Liang Z, Brooks J, Willard M, et al. CXCR4/CXCL12 axis promotes VEGF-mediated tumor angiogenesis through Akt signaling pathway. Biochem Biophys Res Commun 2007;359:716-722.

47. Fabregat I, Malfettone A, Soukupova J. New insights into the crossroads between EMT and stemness in the context of cancer. J Clin Med 2016;5:37. https://doi.org/10.3390/ jcm5030037.

48. Mani SA, Guo W, Liao MJ, et al. The epithelial-mesenchymal transition generates cells with properties of stem cells. Cell 2008;133:704-715.

49. Morel AP, Lievre M, Thomas C, Hinkal G, Ansieau S, Puisieux A. Generation of breast cancer stem cells through epithelial-mesenchymal transition. PLoS One 2008;3:e2888.

50. Rhim AD, Mirek ET, Aiello NM, et al. EMT and dissemination precede pancreatic tumor formation. Cell 2012;148:349-361.

51. Kurrey NK, Jalgaonkar SP, Joglekar AV, et al. Snail and slug mediate radioresistance and chemoresistance by antagonizing p53-mediated apoptosis and acquiring a stem-like phenotype in ovarian cancer cells. Stem Cells 2009;27:2059-2068.

52. Wellner U, Schubert J, Burk UC, et al. The EMT-activator ZEB1 promotes tumorigenicity by repressing stemnessinhibiting microRNAs. Nat Cell Biol 2009;11:1487-1495.

53. Mima K, Okabe $\mathrm{H}$, Ishimoto $\mathrm{T}$, et al. CD44S regulates the 
TGF-beta-mediated mesenchymal phenotype and is associated with poor prognosis in patients with hepatocellular carcinoma. Cancer Res 2012;72:3414-3423.

54. Nomura A, Banerjee S, Chugh R, et al. CD133 initiates tumors, induces epithelial-mesenchymal transition and increases metastasis in pancreatic cancer. Oncotarget 2015;6:8313-8322.

55. Huangyang P, Shang Y. Epigenetic regulation of epithelial to mesenchymal transition. Curr Cancer Drug Targets 2013;13:973-985.

56. Hennessy BT, Gonzalez-Angulo AM, Stemke-Hale K, et al. Characterization of a naturally occurring breast cancer subset enriched in epithelial-to-mesenchymal transition and stem cell characteristics. Cancer Res 2009;69:41164124.

57. Chen Y, Wang K, Qian CN, Leach R. DNA methylation is associated with transcription of snail and slug genes. Biochem Biophys Res Commun 2013;430:1083-1090.

58. Deneberg S, Guardiola P, Lennartsson A, et al. Prognostic DNA methylation patterns in cytogenetically normal acute myeloid leukemia are predefined by stem cell chromatin marks. Blood 2011;118:5573-5582.

59. Chang CJ, Chao CH, Xia W, et al. p53 regulates epithelial-mesenchymal transition and stem cell properties through modulating miRNAs. Nat Cell Biol 2011;13:317323.

6o. Taube JH, Malouf GG, Lu E, et al. Epigenetic silencing of microRNA-203 is required for EMT and cancer stem cell properties. Sci Rep 2013;3:2687.

61. Simonsson S, Gurdon J. DNA demethylation is necessary for the epigenetic reprogramming of somatic cell nuclei. Nat Cell Biol 2004;6:984-990.

62. El Helou R, Wicinski J, Guille A, et al. Brief reports: a distinct DNA methylation signature defines breast cancer stem cells and predicts cancer outcome. Stem Cells 2014;32:3031-3036.

63. Yamazaki Y, Fujita TC, Low EW, Alarcon VB, Yanagimachi R, Marikawa Y. Gradual DNA demethylation of the Oct4 promoter in cloned mouse embryos. Mol Reprod Dev 2006;73:180-188.

64. Liu CC, Lin JH, Hsu TW, et al. IL-6 enriched lung cancer stem-like cell population by inhibition of cell cycle regulators via DNMT1 upregulation. Int J Cancer 2015;136:547559 .

65. Suzuki M, Mohamed S, Nakajima T, et al. Aberrant methylation of CXCL12 in non-small cell lung cancer is associated with an unfavorable prognosis. Int J Oncol 2008;33:113-119.

66. Zhi Y, Chen J, Zhang S, Chang X, Ma J, Dai D. Down-regulation of CXCL12 by DNA hypermethylation and its involvement in gastric cancer metastatic progression. Dig Dis Sci 2012;57:650-659.

67. Fridrichova I, Smolkova B, Kajabova V, et al. CXCL12 and ADAM23 hypermethylation are associated with advanced breast cancers. Transl Res 2015;165:717-730.

68. Kubarek L, Jagodzinski PP. Epigenetic up-regulation of CXCR4 and CXCL12 expression by 17 beta-estradiol and tamoxifen is associated with formation of DNA methyltransferase 3B4 splice variant in Ishikawa endometrial adenocarcinoma cells. FEBS Lett 2007;581:1441-1448.

69. Przybylski M, Kozlowska A, Pietkiewicz PP, Lutkowska A, Lianeri M, Jagodzinski PP. Increased CXCR4 expression in AsPC1 pancreatic carcinoma cells with RNA interference-mediated knockdown of DNMT1 and DNMT3B. Biomed Pharmacother 2010;64:254-258.

70. Koga C, Kobayashi S, Nagano H, et al. Reprogramming using microRNA-302 improves drug sensitivity in hepatocellular carcinoma cells. Ann Surg Oncol 2014;21 Suppl 4:S591-S600.

71. Liu C, Kelnar K, Liu B, et al. The microRNA miR-34a inhibits prostate cancer stem cells and metastasis by directly repressing CD44. Nat Med 2011;17:211-215.

72. Shimono Y, Zabala M, Cho RW, et al. Downregulation of miRNA-20oc links breast cancer stem cells with normal stem cells. Cell 2009;138:592-603.

73. Gwak JM, Kim HJ, Kim EJ, et al. MicroRNA-9 is associated with epithelial-mesenchymal transition, breast cancer stem cell phenotype, and tumor progression in breast cancer. Breast Cancer Res Treat 2014;147:39-49.

74. Yu T, Liu K, Wu Y, et al. MicroRNA-9 inhibits the proliferation of oral squamous cell carcinoma cells by suppressing expression of $\mathrm{CXCR}_{4}$ via the Wnt/beta-catenin signaling pathway. Oncogene 2014;33:5017-5027.

75. Yin P, Peng R, Peng H, et al. MiR-451 suppresses cell proliferation and metastasis in A549 lung cancer cells. Mol Biotechnol 2015:57:1-11.

76. Sarvi S, Mackinnon AC, Avlonitis N, et al. CD133+ cancer stem-like cells in small cell lung cancer are highly tumorigenic and chemoresistant but sensitive to a novel neuropeptide antagonist. Cancer Res 2014;74:1554-1565.

77. Chen Q, Lipkina G, Song Q, Kramer RH. Promoter methylation regulates cadherin switching in squamous cell 
carcinoma. Biochem Biophys Res Commun 2004;315:850856.

78. Peter ME. Let-7 and miR-200 microRNAs: guardians against pluripotency and cancer progression. Cell Cycle 2009;8:843-852.
79. Chen Y, Ramjiawan RR, Reiberger T, et al. CXCR4 inhibition in tumor microenvironment facilitates anti-programmed death receptor-1 immunotherapy in sorafenib-treated hepatocellular carcinoma in mice. Hepatology 2015;61:1591-1602. 